\title{
Efeito de Níveis de Cálcio da Dieta de Filhotes de Ema (Rhea americana, Linnaeus, 1758), na Prevenção do Encurvamento de Pernas, em Cativeiro
}

\author{
Marisa E. Sanchez Bastos ${ }^{2}$, Helder Louvandini ${ }^{3}$, Marcia Nogueira ${ }^{4}$, Cristian A. Carranza ${ }^{5}$, \\ Abel Lavorenti ${ }^{4}$
}

\begin{abstract}
RESUMO -O objetivo deste trabalho foi avaliar dois níveis de cálcio $(0,7$ e $1,4 \%)$ na dieta de filhotes de ema (Rhea americana). Doze filhotes de ema com 30 dias de idade foram distribuídas, em pares, em seis piquetes com $100 \mathrm{~m}^{2}$, em delineamento experimental inteiramente casualizado com três repetições. O consumo foi avaliado diariamente e o peso e as características de crescimento (medidas do comprimento do bico, do osso tibiotarso e metatarso) foram avaliadas semanalmente, durante 60 dias. Os filhotes de ema mantidos na área de $100 \mathrm{~m}^{2}$ de capim e terra, que se deslocaram normalmente, não mostraram entortamento em nenhum dos níveis de cálcio fornecidos, mas as tratadas com dietas contendo $1,4 \%$ de cálcio apresentaram melhores pesos e características de crescimento em relação às que receberam dietas com $0,7 \%$ de cálcio.
\end{abstract}

Palavras-chave: cálcio, ema, encurvamento de pernas

\section{Effect of Calcium Levels in Diet of Chicken Rhea (Rhea americana, Linnaeus, 1758), to Prevent Bow Leg Syndrome, in Captivity}

\begin{abstract}
The objective of this work was to evaluate two levels of calcium (.7 and 1.4\%) in the diet of chicken Rhea (Rhea americana). Twelve chicken Rhea with 30 days of age were allotted in pare, in six plots with $100 \mathrm{~m}^{2}$, in a completely randomized experiment design with three replications. The feed intake was daily evaluated and the weight and the growth characteristics (length of beak, tibiotarsus and metatarsus measurements) were weekly evaluated, during 60 days. The chicken Rhea maintained in a $100 \mathrm{~m}^{2}$ of grass and nude soil, that normally dislocated, did not show bow leg syndrome in anyone of the calcium level fed, but those treated with diets containing $1.4 \%$ of calcium presented better weight and growth characteristics than those that received diet with $.7 \%$ of calcium.

Key Words: calcium, Rhea, bow leg syndrome
\end{abstract}

\section{Introdução}

Existe atualmente na Europa, na Índia, no Canadá, nos Estados Unidos, no Uruguai, no Brasil e na Argentina grande interesse na criação da ema (Rhea america$n a$, Linnaeus, 1758) em cativeiro com o objetivo de explorá-la economicamente. A incidência de desordens no sistema locomotor nestas aves é uma das principais causas de mortalidade, sendo o período mais crítico até os três meses de idade (CHAPMAN e BASS, 1994; FEZLER e BEAL, 1996) na criação intensiva. O osso é um tecido muito complexo que está intimamente relacionado com o crescimento em geral. Vários nutrientes influenciam o crescimento, o desenvolvimento e a manutenção óssea dos animais. O desbalanceamento no metabolismo de cálcio $(\mathrm{Ca})$ e o rápido ganho de peso são as principais causas de encurvamento dos ossos nas emas (GUITTIN, 1986). O cálcio deve manter com o fósforo $(\mathrm{P})$ relação, em torno de 2:1, na dieta das emas, para se evitar a desmineralização (COIMBRA-FILHO e CRUZ ROCHA, 1973; FLIEG, 1973; e Perelman, citado por JENSEN et al., 1992). Além das deficiências nutricionais, o entortamento das pernas também é atribuído à falta de exercício (DOLENSEK e BRUNNING, 1986; JENSEN, 1989; JENSEN et al., 1992; e WADE, 1992). O objetivo deste trabalho foi testar diferentes níveis de cálcio na dieta, segundo as exigências nutricionais dos filhotes de ema, e analisar a capacidade de deslocamento dos animais no recinto, estabelecendo sua relação com o entortamento.

\footnotetext{
${ }^{1}$ Parte da Dissertação de Mestrado do primeiro autor apresentada à USP/ESALQ, Piracicaba-SP

2 Bolsista da CAPES.

3 Departamento de Medicina Veterinaria Preventiva e Saúde Animal USP/FMVZ, SP.

${ }^{4}$ Departamento de Zootecnia-USP/ESALQ, Piracicaba-SP.

${ }^{5}$ Departamento de Matemática e Estatística-USP/ESALQ, Piracicaba-SP
} 


\section{Materiais e Métodos}

O experimento (com Auxílio da Pesquisa da Fundação de Amparo à Pesquisa do Estado de São Paulo -FAPESP- Processo $n^{0}$ 1994/3935-8) foi conduzido nas instalações da Comissão de Investigação em Zootecnia e Biologia de Animais Silvestres (CIZBAS), do Departamento de Zootecnia da Escola de Agronomia "Luiz de Queiroz", Universidade de São Paulo (ESALQ/USP) de Piracicaba- SP. Foram utilizados 12 filhotes de ema com 30 dias de idade, alojados em seis recintos de $100 \mathrm{~m}^{2}$ (dois animais por recinto), com área coberta de $10,21 \mathrm{~m}^{2}$, chão de cimento, onde foi colocada gaiola com capim seco, para os animais dormirem à noite. Antes do início do experimento, foram feitas coletas de material fecal, para o controle parasitológico, por meio do método de flutuação simples da técnica de Willis (WILLIS, 1927), quando se utilizou o vermífugo para aves domésticas "Proverme" (citrato de piperazina tetrahidratado), repetida após 15 dias.

Durante 60 dias, os animais foram alimentados com dois tipos de rações (Tabela 1), uma contendo $0,7 \%$ de $\mathrm{Ca}$ (tratamento 1 , relação $\mathrm{Ca}: \mathrm{P} \quad 1: 1$ ) e a outra com 1,4\% de Ca (tratamento 2, relação $\mathrm{Ca}: \mathrm{P}$ $2: 1$ ), resultando seis animais por tratamento.

No período experimental, verificou-se consumo diário da ração e, semanalmente, foram avaliados o peso e alguns parâmetros de crescimento (medidas do comprimento do bico, comprimento do osso tibiotarso e do osso metatarso).

A medição do deslocamento dos animais dentro do recinto foi medido por intermédio do Método de Amostragem "Scan Sampling" (MARTIN e BATESON, 1986), que consiste em observar o animal, descrevendo sua atividade nos intervalos estabelecidos. As observações foram feitas a cada cinco minutos, distribuídos no horário das $8 \mathrm{~h} 30$ às $19 \mathrm{~h}$, aproximadamente, a quatro metros de distância dos piquetes. Estas observações permitiram conhecer as atividades diárias realizadas pelos filhotes, avaliando, dessa forma, o exercício. O comportamento foi usado como variável categórica, com 11 pautas comportamentais. A partir das contagens ao longo do tempo estabelecido como de amostragens, foram obtidas as porcentagens como medidas descritivas para cada pauta comportamental.

No final do experimento, os animais foram sexados por intermédio da técnica de reversão de cloaca (ANON, 1995). O sangue foi colhido da veia radial para determinação do Hematócrito por meio da cen- trífuga de microhematócrito, Hemoglobina com fita de papel Hawksley Haemoglobin Scale. Após a centrifugação, foi obtido o plasma para determinação do fósforo, segundo a técnica de FISKE e SUBBAROW (1925). Com finalidade de verificar a mineralização óssea, foi realizada radiografias dos ossos tibiotarso e metatarso dos filhotes, sedando as aves. Verificando-se excitação inicial, foi necessário realizar associação com outro sedativo (JENSEN et al., 1992), utilizando cloridrato de cetamina (Ketalar), para diminuir o estresse durante o transporte dos filhotes e facilitar a contenção dos mesmos, na exposição ao raio $\mathrm{X}$.

Foi utilizado delineamento experimental inteiramente casualizado, com dois tratamentos e três repetições, em que cada repetição possuía dois animais, num total de 12 animais. As análises da variância para

\begin{tabular}{|c|c|c|}
\hline \multirow[t]{2}{*}{$\begin{array}{l}\text { Ingrediente } \\
\text { Ingredient }\end{array}$} & \multicolumn{2}{|c|}{$\begin{array}{c}\text { Tratamento } \\
\text { Treatment }\end{array}$} \\
\hline & 1 & 2 \\
\hline Milho & 34,94 & 34,94 \\
\hline Corn & & \\
\hline $\begin{array}{l}\text { Farelo de trigo } \\
\text { Wheat meal }\end{array}$ & 29,70 & 29,70 \\
\hline $\begin{array}{l}\text { Farelo de soja } \\
\text { Soybean meal }\end{array}$ & 20,70 & 20,70 \\
\hline $\begin{array}{l}\text { Farinha de carne } \\
\text { Meat meal }\end{array}$ & 2,51 & 2,51 \\
\hline $\begin{array}{l}\text { Calcário } \\
\text { Linestone }\end{array}$ & 0,70 & 2,60 \\
\hline $\begin{array}{l}\text { Casca de arroz } \\
\text { Rice hulls }\end{array}$ & 4,90 & 4,90 \\
\hline $\begin{array}{l}\text { Óleo vegetal } \\
\text { Vegetable oil }\end{array}$ & 0,50 & 0,50 \\
\hline Sal & 0,45 & 0,45 \\
\hline Salt & & \\
\hline Caulim & 5,10 & 3,20 \\
\hline $\begin{array}{l}\text { Premix mineral e vitamínico } \\
\text { Mineral-vitamin premix }\end{array}$ & 0,50 & 0,50 \\
\hline $\begin{array}{l}\mathrm{EM}(\mathrm{kcal} / \mathrm{kg}) \\
M E\end{array}$ & 2235 & 2235 \\
\hline $\mathrm{PB}(C P)$ & 18,3 & 18,3 \\
\hline $\mathrm{FB}(C F)$ & 6,85 & 6,85 \\
\hline $\mathrm{EE}$ & 6,80 & 6,80 \\
\hline $\mathrm{Ca}$ & 0,7 & 1,4 \\
\hline$\underline{\mathrm{P}}$ & 0,6 & 0,6 \\
\hline $\begin{array}{l}1 \text { 1Premix mineral/kg de ração (Pre } \\
\text { (Manganese), } 80 \text { mg; Zinco (Zinc), } \\
\text { (Copper), } 30 \mathrm{mg} \text {; lodo (Iodine), } 4 \mathrm{mg} \\
\text { (Cobalt), } 4 \mathrm{mg} \text {. Premix vitamínico/k } \\
\text { Vit. A, } 12.000 \text { Ul; Vit. } \mathrm{D}_{3}, 1500 \mathrm{U} \\
\mathrm{B}_{1}, 12 \mathrm{mg} ; \text { Vit. } \mathrm{B}_{6}, 4 \mathrm{mg} ; \text { Pantoten } \\
15 \mathrm{mg} ; \text { Biotina, } 0,1 \mathrm{mg} ; \text { Vit. } \mathrm{K}_{3}, 4 \mathrm{n} \\
\text { Metionina (Methionine), } 100 \mathrm{mg} \text {; Li } \\
\text { Vit. } \mathrm{B}_{12}, 20 \text { microgramas; Niacina } \\
100 \mathrm{mg} \text {. }\end{array}$ & $\begin{array}{l}\text { ix mineral/ } \\
\text { mg; Ferr } \\
\text { Selênio }(S \\
\text { le ração }( \\
\text { Vit. } \mathrm{B}_{2}, 5 \\
\text { de cálcio } \\
\text {; Ácido fó } \\
\text { la (Lysine) } \\
\text { Niacin), } 20\end{array}$ & $\begin{array}{l}\text { Manganês } \\
\text { mg; Cobre } \\
1 \mathrm{~g} \text {;Cobalto } \\
\text { in/kg of diet): } \\
22 \mathrm{mg} \text {; Vit. } \\
\text { ntothenate), } \\
\text { id), } 0,6 \mathrm{mg} \text {; } \\
\text { HT, } 10 \mathrm{mg} \text {; } \\
\text { a (Choline), }\end{array}$ \\
\hline
\end{tabular}


as variáveis de resposta, peso do animal, consumo, ganho de peso e medidas de crescimento, foram realizadas pelo SAS e pelas análises de comportamento por porcentagens, com planilha eletrônica convencional.

\section{Resultados e Discussão}

No exame parasitológico, apenas um animal apresentou numerosas formas ovaladas, características de ovos de Helmintos, sendo que após a vermifugação o animal apresentou-se negativo. As radiografias não evidenciaram a falta de mineralização óssea dos filhotes submetidos às dietas testadas. Observou-se, por intermédio da sexagem, número igual de machos e fêmeas. As coletas de sangue foram feitas na veia radial da asa das aves, embora a coleta na veia do metatarso permitisse contenção mais confortável. Não houve efeito significativo nos níveis de hematócrito e hemoglobina com relação ao sexo do filhote. Embora não tenham sido observadas diferenças significativas verificou-se tendência para valores de hematócrito serem maiores nas fêmeas em relação aos machos, nos dois tratamentos (Tabela 2). Os valores obtidos neste trabalho foram semelhantes aos obtidos nos trabalhos de JUNCKER (1993), que observou valores para hematócrito e hemoglobina de 30,4 e 12,16\%, respectivamente. JENSEN et al. (1992) observaram em filhotes de avestruz valores entre $10 \mathrm{e}$ $20 \%$ de hemoglobina, com média observada de $12,2 \%$.

Os valores de fósforo obtidos no plasma são apresentados na Tabela 2. Não houve efeito significativo nos níveis de fósforo com relação ao sexo dos filhotes. Os valores observados por Perelman, citado por JENSEN et al. (1992), foram de 5,23 $\mathrm{mg} / \%$ para o avestruz e 3,66 mg/\% para o emu. OKOTIEEBOH et al. (1992) obtiveram $5,2 \mathrm{mg} / \%$ para o avestruz e 5,4 mg/\% para o emu. Os valores mínimos e máximos observados por Hodges, citado por JENSEN et al. (1992), foram de 4,0 a 9,9\% para os avestruzes. Estes valores sugerem que os valores obtidos para os filhotes de ema neste trabalho estão dentro dos parâmetros observados para outras espécies do grupo das Ratitas.

As medições feitas para avaliar o exercício diário nos filhotes em cativeiro permitiram observar as tendências de comportamento individual. Foram obtidas as porcentagens como medidas descritivas para cada pauta comportamental, observando que a maior parte do tempo as emas se encontravam caminhando, $44,29 \%$, e bicando o chão (comportamento exploratório), 33,70\%. Este fato pode ser atribuído à natureza das emas que andam ininterruptamente (SICK, 1984). DEGEN et al. (1989) observaram que a maior atividade dos filhotes de avestruz, em cativeiro, é o caminhar (61,5\%). Pode-se inferir que a área de $100 \mathrm{~m}^{2}$ foi suficiente para o animal se exercitar normalmente. Na Tabela 3 são apresentados os valores médios dos parâmetros avaliados (comprimento do bico, do osso tibiotarso e metatarso), que indicaram valores diferentes $(\mathrm{P}<0,0001)$ entre os tratamentos, sendo maiores no tratamento 2, contendo $1,4 \%$ de $\mathrm{Ca}$ e com melhor proporção de $\mathrm{Ca}: \mathrm{P}(2: 1)$.

Para estudar o comportamento multidimensional do crescimento dos filhotes, os dados referentes aos comprimentos do bico, do tibiotarso e do metatarso foram submetidos à análise de correlação, mostrando os valores estimados para os coeficientes de correlação. Pode-se observar que as variáveis peso, comprimento do bico, tibiotarso e metatarso estão correlacionadas, ou seja, estes parâmetros estão altamente associados. Foi efetuada a análise de regressão das variáveis, ao longo do período experimental, observando-se que a taxa de aumento de peso, em função do consumo (Figura 1), o compri-

Tabela 2 - Valores médios e desvio-padrão obtidos para hemoglobina $(\mathrm{Hb})$ e hematócrito (Htc), em sangue, e fósforo no plasma, para cada sexo e dieta, em filhotes de ema com aproximadamente 90 dias de idade

Table 2 - Mean and standard deviation of hemoglobin $(\mathrm{Hb})$ and hematocrit (PCV) in blood and phosphorus in plasma for each sex and diet, in chicken Rhea with nearly 90 days of age

\begin{tabular}{|c|c|c|c|c|}
\hline \multirow[t]{3}{*}{$\begin{array}{l}\text { Parâmetro } \\
\text { Parameter }\end{array}$} & $\begin{array}{l}\text { Macho } \\
\text { Male }\end{array}$ & $\begin{array}{c}\text { Macho } \\
\text { Male }\end{array}$ & $\begin{array}{l}\text { Fêmea } \\
\text { Female }\end{array}$ & $\begin{array}{l}\text { Fêmea } \\
\text { Female }\end{array}$ \\
\hline & \multicolumn{2}{|c|}{$\begin{array}{c}\text { Tratamento } \\
\text { Treatment }\end{array}$} & \multicolumn{2}{|c|}{$\begin{array}{c}\text { Tratamento } \\
\text { Treatment }\end{array}$} \\
\hline & 1 & 2 & 1 & 2 \\
\hline$\overline{\mathrm{Hb}(\mathrm{g} / \%)}$ & $12,48 \pm 2,21$ & $12,48 \pm 2,38$ & $12,48 \pm 1,80$ & $14,85 \pm 1,10$ \\
\hline $\begin{array}{l}\mathrm{Htc}(\%) \\
P C V\end{array}$ & $33,00 \pm 1,41$ & $33,50 \pm 8,50$ & $36,25 \pm 3,40$ & $37,50 \pm 4,95$ \\
\hline $\mathrm{P}(\mathrm{mg} / \%)$ & $4,93 \pm 1,10$ & $5,19 \pm 1,43$ & $4,99 \pm 1,58$ & $3,94 \pm 0,87$ \\
\hline
\end{tabular}


BASTOS et al.

Tabela 3 - Análise descritiva dos valores médios, das variáveis estudadas semanalmente para os tratamentos Table 3 - Descriptive analysis of mean values, of the variables weekly studied for the treatments

\begin{tabular}{|c|c|c|c|c|c|c|c|}
\hline \multirow[t]{3}{*}{$\begin{array}{l}\text { Variável } \\
\text { Variable }\end{array}$} & \multicolumn{4}{|c|}{$\begin{array}{c}\text { Tratamento } 1 \\
\text { Treatment }\end{array}$} & \multicolumn{3}{|c|}{$\begin{array}{c}\text { Tratamento } 2 \\
\text { Treatment }\end{array}$} \\
\hline & \multirow[t]{2}{*}{$\mathrm{CV}$} & \multirow{2}{*}{$\begin{array}{l}\text { Média } \pm \text { erro-padrão } \\
\text { Mean } \pm \text { standard error }\end{array}$} & \multicolumn{2}{|c|}{$\begin{array}{l}\mathrm{IC} \\
C I \\
\end{array}$} & \multirow{2}{*}{$\begin{array}{l}\text { Média } \pm \text { erro-padrão } \\
\text { Mean } \pm \text { standard error }\end{array}$} & \multicolumn{2}{|c|}{$\begin{array}{l}\mathrm{IC} \\
C I\end{array}$} \\
\hline & & & $\begin{array}{l}\text { Inf. } \\
\text { Lower }\end{array}$ & $\begin{array}{l}\text { Sup. } \\
\text { Upper }\end{array}$ & & $\begin{array}{l}\text { Inf. } \\
\text { Lower }\end{array}$ & $\begin{array}{l}\text { Sup. } \\
\text { Upper }\end{array}$ \\
\hline $\begin{array}{l}\text { Peso (g) } \\
\text { Weight }\end{array}$ & 45,60 & $1459 \pm 644$ & 1272 & 1647 & $2213 \pm 828$ & 2007 & 2418 \\
\hline $\begin{array}{l}\text { Consumo }(\mathrm{g}) \\
\text { Intake }\end{array}$ & 48,95 & $576 \pm 275$ & 496 & 656 & $790 \pm 354$ & 703 & 878 \\
\hline $\begin{array}{l}\text { C. bico }(\mathrm{cm}) \\
\text { Beaklength }\end{array}$ & 8,96 & $6,7 \pm 0,6$ & 6,6 & 6,9 & $7,3 \pm 0,5$ & 7,2 & 7,5 \\
\hline $\begin{array}{l}\text { C.tibiotarso }(\mathrm{cm}) \\
\text { Tibiotarsus length }\end{array}$ & 18,42 & $16,9 \pm 3,2$ & 16,1 & 17,7 & $20,0 \pm 2,8$ & 19,1 & 20,9 \\
\hline $\begin{array}{l}\text { C.metatarso }(\mathrm{cm}) \\
\text { Metatarsus length }\end{array}$ & 17,96 & $13,7 \pm 2,4$ & 13,1 & 14,4 & $16,1 \pm 2,4$ & 15,4 & 16,8 \\
\hline
\end{tabular}

mento do tibiotarso e o comprimento do metatarso foram maiores no tratamento 2. Na Figura 2 observase que os ossos tibiotarso e o metatarso cresceram proporcionalmente em comprimento. Estas informações indicam que as características morfológicas medidas (comprimento do bico, tibiotarso e metatarso) podem ser utilizadas na seleção indireta. A variável ganho de peso apresentou comportamento melhor no tratamento 2 $(2,192 \pm 397,96)$, obtendo-se média menor no tratamento $1(1,455 \pm 459,35)$ que no tratamento $2(\mathrm{P}=0,01)$.

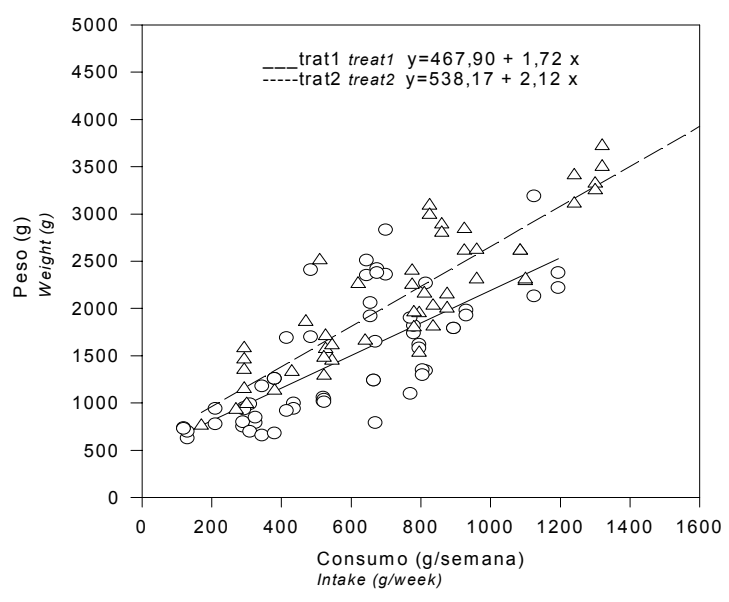

Figura 1 - Regressão linear do consumo semanal. Figure 1- Linear regression of weekly intake.

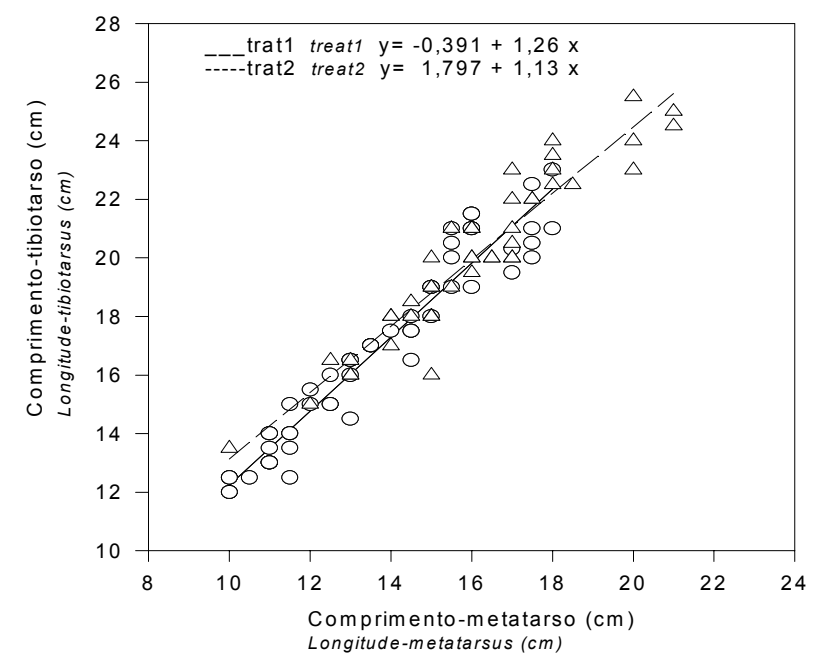

Figura 2 - Regressão linear do crescimento proporcional dos ossos tibiotarso e metatarso.

Figure 2- Linear regression proportional growth of tibiotarsus and metatarsus bones.

\section{Conclusões}

Nas condições em que foi realizado o presente trabalho e para o grupo estudado, pode-se concluir que o nível de $1,4 \%$ de cálcio e a relação cálcio:fósforo 2:1 proporcionaram melhores pesos e parâmetros de crescimento, em filhotes de ema de 30 a 90 dias de idade. Não foram observados casos de encurvamento de pernas nos filhotes, os quais conseguiram se deslocar normalmente. 


\section{Agradecimentos}

À Fundação Coordenação de Aperfeiçoamento de Pessoal de Nível Superior (CAPES), pela bolsa concedida. À Fundação de Amparo à Pesquisa do Estado de São Paulo (FAPESP), pelo auxílio da pesquisa. À Supre-Mais Produtos Bioquímicos Ltda. de Valinhos-SP, pelo fornecimento e balanceamento dos ingredientes das rações. Ao Parque Ecológico de São Carlos-SP, pelo empréstimo dos animais.

\section{Referências Bibliográficas}

ANON. DNA sexing. Canadian Ostrich, 4(2):20, 1995. Resumo em Ladd, k. (comp.). Ratites bibliographys.

CHAPMAN, H., BASS, F. 1994. Modern concepts for commercial Rhea Prodution. Palm City: Porter Professional Publishers. $83 \mathrm{p}$.

COIMBRA FILHO, A., CRUZ ROCHA, N. 1973. Aspectos do processo nutricional de animais selvagens em cativeiro. Brasil Florestal, 4(14):19-35.

DEGEN, A.A., KAM, M., ROSENSTRAUCH, A. 1989. Time activity budget of ostriches (Struthio camelus) offered concentrate feed and maintained in outdoor pens. Applied Anim. Behaviour Sci., 22(3/4):347-358.

DOLENSEK, E., BRUNING, D. 1986. Ratites (Struthioniformes, Casuariiformes, Rheiformes, Tinamiformes, and Apterygiformes). In: FOWLER, M. Zoo \& wild animal medicine. Philadelphia: W.B.Saunders. cap.22. p.276-292.

FEZLER, D., BEAL, D. 1996. Rubber rhea syndrome and fading chick syndrome in rheas and ostriches. In: The Rhea Report. The official Publication of the North American Rhea Association. 3(1):16-21.

FISKE, C.H., SUBBAROW, Y. 1925. The colorimetric determination of phosphorus. J. Biol. Chem., 66(2):375-400.
FLIEG, G.M. 1973. Nutritional problems in young Ratites. International Zoo Yearbook, 3:158-163.

GUITTIN, P. 1986. Bow leg syndrome in Ratites birds. Avicultural Magazine, 92(2):71-79.

JENSEN, J. 1989. Husbandry, medical and surgical management of ratites: part I. Proceeding of the American Association of Zoo Veterinarians, p.113-118.

JENSEN, J., JOHNSON, J.H., WEINER, S.T. 1992. Husbandry and medical management of ostriches, emus and rheas. S.L.: Wildlife and Exotic Animal TeleConsultants. 129p.

JUNCKER, T. 1993. Little creek ostrich ranch (Separata de MULTISTATE BIG BIRD CONFERENCE, Indianapoli. p.1-5.)

MARTIN, P., BATESSON, P. 1986. Measuring behaviour. Washington: FRS. 200p.

OKOTIE-EBOH, G., BAILEY C.A., HICKS, K.D. et al. 1992. Reference serum biochemical values for emus and ostriches. Amer. J. Vet. Res., 53(10):1765-1768.

SICK, H. 1984. Ornitología brasileira: uma introdução. 3. ed. Brasília: Universidade de Brasília. 827p.

STATISTICAL ANALYSIS SYSTEM. SAS System for linear models. Cary: SAS Institute, 1986. 211p.

WADE, J.R. Ratites pediatric medicine and surgery. 1992. Proceedings of the Assoc. Avian Vet., p. 340-353.

WILLIS, H.H. 1927. A simple levetation method for the detection of hook worma ova. Med. J. Austr., 8:375-376.

Recebido em: 23/10/97

Aceito em: 04/08/98 